\title{
Solar cycle variations in polar cap area measured by the superDARN radars
}

\author{
S. M. Imber, ${ }^{1}$ S. E. Milan, ${ }^{1}$ and M. Lester ${ }^{1}$ \\ Received 12 December 2012; revised 9 August 2013; accepted 10 August 2013; published 4 October 2013.
}

[1] We present a long-term study, from January 1996 to August 2012, of the latitude of the Heppner-Maynard Boundary (HMB) measured at midnight using the northern hemisphere Super Dual Auroral Radar Network (SuperDARN). The HMB represents the equatorward extent of ionospheric convection and is used in this study as a measure of the global magnetospheric dynamics. We find that the yearly distribution of HMB latitudes is single peaked at $64^{\circ}$ magnetic latitude for the majority of the 17 year interval. During 2003, the envelope of the distribution shifts to lower latitudes and a second peak in the distribution is observed at $61^{\circ}$. The solar wind-magnetosphere coupling function derived by Milan et al. (2012) suggests that the solar wind driving during this year was significantly higher than during the rest of the 17 year interval. In contrast, during the period 2008-2011, HMB distribution shifts to higher latitudes, and a second peak in the distribution is again observed, this time at $68^{\circ}$ magnetic latitude. This time interval corresponds to a period of extremely low solar wind driving during the recent extreme solar minimum. This is the first long-term study of the polar cap area and the results demonstrate that there is a close relationship between the solar activity cycle and the area of the polar cap on a large-scale, statistical basis.

Citation: Imber, S. M., S. E. Milan, and M. Lester (2013), Solar cycle variations in polar cap area measured by the superDARN radars, J. Geophys. Res. Space Physics, 118, 6188-6196, doi:10.1002/jgra.50509.

\section{Introduction}

[2] The cycling of magnetic flux through the Earth's magnetosphere is largely controlled by magnetic reconnection [Dungey, 1961; Siscoe and Huang, 1985; Cowley and Lockwood 1992]. Reconnection at low latitudes on the dayside magnetopause takes place whenever the interplanetary magnetic field (IMF) has a southward component and leads to a buildup of magnetic flux in the magnetotail. The resulting high magnetic pressure in the tail is relieved by reconnection between the tail lobes, closing lobe field lines and accelerating plasma Earthward and tailward. This cycle typically repeats every few hours and is known as the substorm cycle [e.g., Akasofu, 1964; McPherron et al., 1973; Hones, 1979; Pulkkinen et al., 1991; Lockwood and Cowley, 1992; Tanskanen, 2009]. The polar cap is the region of open field lines with footprints at high latitudes in both hemispheres, and its equatorward edge is known as the open/closed field line boundary (OCB). The cycling of magnetic flux during substorms drives a characteristic ionospheric flow pattern as the system adjusts to changes in the open flux content of the magnetosphere as shown in

\footnotetext{
${ }^{1}$ Department of Physics and Astronomy, University of Leicester, Leicester, UK.

Corresponding author: S. M. Imber, Department of Physics and Astronomy, University of Leicester, University Road, Leicester LE1 7RH, UK. (si45@ion.le.ac.uk)

(C)2013. American Geophysical Union. All Rights Reserved. 2169-9380/13/10.1002/jgra.50509
}

Figure 1 [e.g., Cowley and Lockwood, 1992]. The left panel shows the ionospheric flows for low latitude dayside reconnection, and the right panel is the tail reconnection case. In each panel, the solid black circle marks the open/closed field line boundary and the dashed lines are the merging gaps - the ionospheric projection of the reconnection sites. Away from the merging gaps, the flow is adiaroic so is moving with, and not crossing, the boundary. The black arrowed lines show the flow streamlines in the ionosphere, forming a twin cell convection pattern with antisunward flow across the polar cap at high latitudes and return flow at lower latitudes. Whether the polar cap is expanding or contracting at a given time depends upon the relative rates of reconnection on the dayside and in the tail, as auroral observations have shown that the polar cap shrinks and expands according to the amount of open flux in the system [Cowley and Lockwood, 1992; Taylor et al., 1996; Milan et al., 2004, 2007]. The rate of change of the polar cap area at a given time is explained by the expanding-contracting polar cap paradigm (ECPC), which is based upon the following form of Faraday's Law:

$$
\frac{d F_{P C}}{d t}=\Phi_{D}-\Phi_{N}=\frac{d}{d t} \int_{P C} \mathbf{B} . \mathbf{d S}
$$

where $\mathrm{F}_{\mathrm{PC}}$ is the open magnetic flux in the polar cap, $\Phi_{D}$ is the dayside reconnection rate, $\Phi_{N}$ is the reconnection rate in the tail, $\mathbf{B}$ is the ionospheric magnetic field vector, and the integral is taken over the polar cap area. An expanding polar cap area suggests that low latitude dayside reconnection dominates over reconnection in the tail, and the opposite applies for a contracting polar cap. The polar 

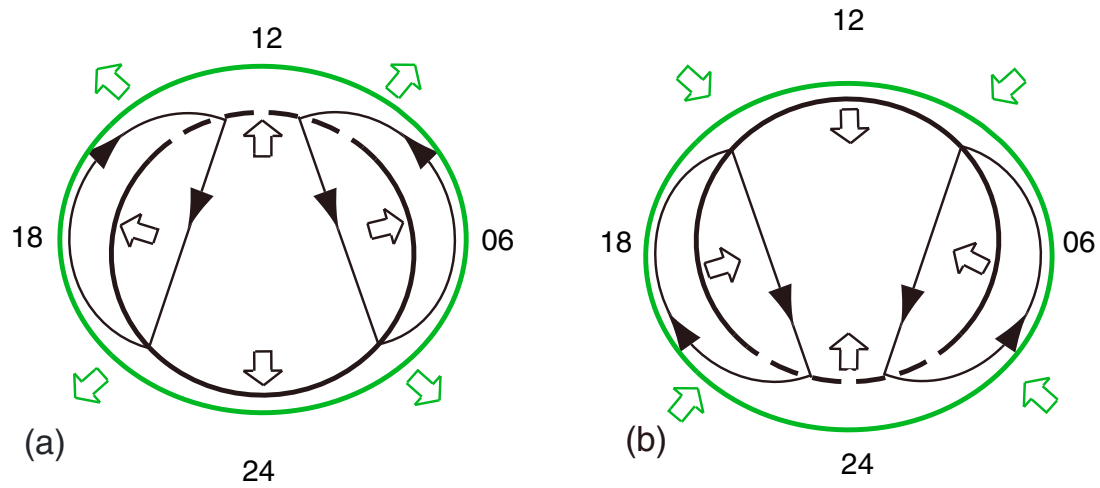

\begin{abstract}
Figure 1. A schematic to show the ionospheric convection pattern driven by (a) low latitude dayside reconnection and (b) tail reconnection. Noon is to the top of each panel, with dusk to the left. The black circle represents the open/closed field line boundary (OCB) which encircles the open field line region. The dashed portion of this circle is the ionospheric projection of the reconnection site, known as the merging gap. The black arrowed lines show the direction of the ionospheric plasma flow which crosses the OCB at the merging gap in both cases. The white arrows represent the expansion or contraction of the polar cap. The green line marks the Heppner-Maynard Boundary (HMB), with green arrows showing the motion of the HMB as the polar cap expands or contracts.
\end{abstract}

cap area is only stable for extended periods when dayside and nightside reconnection are exactly balanced - a phenomenon known as steady magnetospheric convection. More commonly, the substorm cycle of expansion followed by contraction of the polar cap is observed, driving fast ionospheric flows and also leading to a brightening of the aurora [e.g., Milan et al., 2007].

[3] While the reconnection rate in the tail can be extremely difficult to measure, many studies have related the dayside reconnection rate to the upstream solar wind conditions via coupling functions. An early example of a coupling function is that of Kan and Lee [1979]:

$$
\Phi=\mathbf{B}_{S} V_{S} \sin ^{2}\left({ }^{\theta} / 2\right) l_{0}
$$

where $\Phi$ is the polar cap potential, $\mathbf{B}_{\mathrm{S}}$ is the southward component of the IMF (zero for northward IMF), $\mathrm{V}_{\mathrm{S}}$ is the plasma inflow speed, $\Theta$ is the clock angle, and $l_{0}$ is a constant, $7 \mathrm{R}_{\mathrm{E}}$, representing the effective length of the reconnection site.

[4] Subsequent studies have refined these early coupling functions using upstream solar wind measurements in combination with ground-based instrumentation, auroral images, and in situ spacecraft measurements [e.g., Siscoe et al., 2002; Newell et al., 2007; Borovsky, 2008, Milan et al., 2012]. The coupling function used in this study is that of Milan et al. [2012]:

$$
\begin{gathered}
\Phi_{D}=L_{e f f} V_{X} B_{Y Z} \sin ^{9 / 2}(\theta / 2) \\
L_{\text {eff }}=3.8 R_{E}\left(\frac{V_{X}}{4 \times 10^{5} m s^{-1}}\right)^{1 / 3}
\end{gathered}
$$

where $\Phi_{D}$ is the dayside reconnection rate, $V_{X}$ is the velocity of the solar wind in GSM coordinates, $\mathrm{L}_{\mathrm{eff}}$ is the effective length of the reconnection site modulated by the solar wind velocity, $\mathrm{B}_{\mathrm{YZ}}$ is the transverse component of the $\mathrm{IMF}\left(B_{Y Z}^{2}=\right.$ $B_{y}^{2}+B_{z}^{2}$ ), and $\Theta$ is the clock angle.

[5] The location of the open/closed field line boundary (OCB) is an extremely valuable tool when considering large-scale magnetospheric dynamics. There are two commonly used techniques to determine the location of the OCB: global auroral imagery and in situ plasma observations. Satellites in high inclination polar orbits are able to measure the sharp change in plasma parameters when the spacecraft passes from the closed field line region to open field lines [e.g., Newell et al., 1991]. The advantage of this technique is that the boundary can often be identified with high accuracy, although it is a point measurement at a single time and therefore cannot give information about the total area of the polar cap or its evolution over time scales of tens of minutes. Global auroral images are also used to obtain the location and motion of the OCB [e.g., Hubert et al., 2006, 2010; Boakes et al., 2008; Milan et al., 2009a, 2009b; Longden et al., 2010]. The accuracy of the OCB measurement depends on the resolution of the camera, the viewing angle, dayglow, and the accuracy of the relation between the auroral features and the OCB itself. Such observations have the advantage of continuous coverage of the entire auroral oval for extended time periods of many hours such that the large-scale motion of the OCB can be observed. While auroral imaging missions have been highly successful in providing information about the magnetosphere [e.g., Ostgaard et al., 2007; Milan et al. 2008, 2009a, 2009b; Boakes et al., 2009; Hubert et al., 2010], high quality auroral images have only been available for a few years during the last decade, and since 2008 no auroral images have been available whatsoever.

[6] While the OCB encloses the region of open field lines, the ionospheric convection pattern frequently extends to lower latitudes as the return flow of the twin cell convection pattern is on closed field lines. The green circle in each panel of Figure 1 represents the latitude of the Heppner-Maynard Boundary, below which the flow is dominated by corotation, and above which it is driven by convection. The characteristic shape of the boundary (roughly circular on the nightside, moving to higher latitudes on the dayside) was determined empirically using electric field data from dawn-dusk OGO 6 passes [Heppner, 1977], and later these models were 


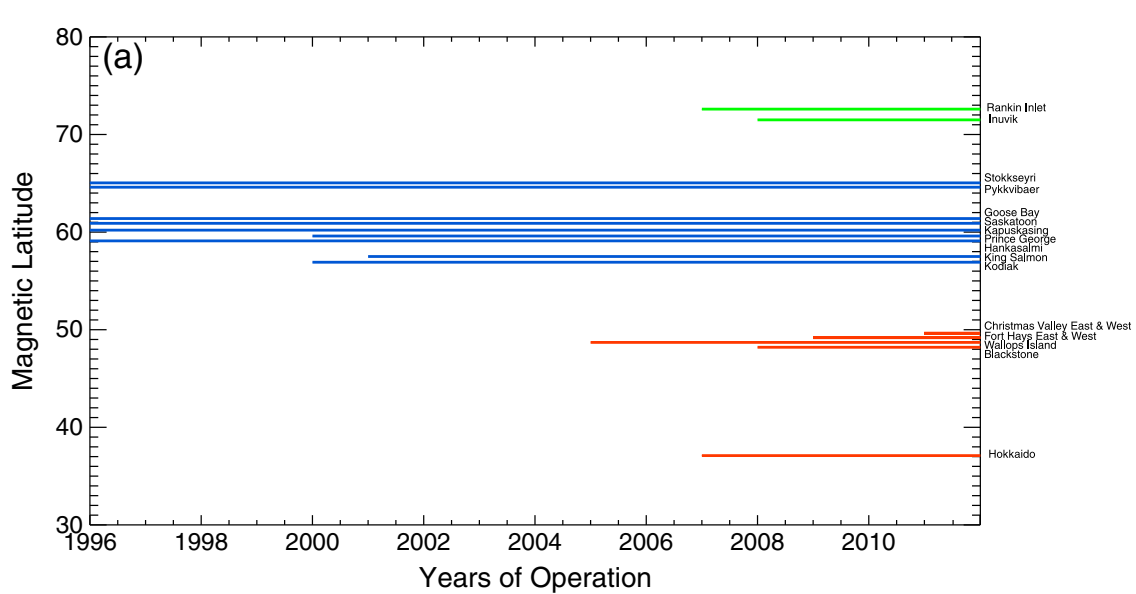

(b)

Figure 2. (a) The years of operation of each of the northern hemisphere Super Dual Auroral Radar Network (SuperDARN) radars are shown by the colored bars, ordered by their geomagnetic latitude. The PolarDARN radars are shown in green, the main array in blue, and the StormDARN radars in red. (b) The fields of view of all northern hemisphere radars operational on 31 December 2011, at 00 UT. The colors of the fields of view match those in Figure $2 \mathrm{a}$.

refined using Dynamics Explorer 2 (DE2) electric field data [Heppner and Maynard, 1987].

[7] In a previous study, Imber et al. [2013] have shown that on a large-scale, statistical basis when the Super Dual Auroral Radar Network (SuperDARN) data coverage is good, the HMB may be used as a proxy for the OCB with an average offset between the two boundaries of $7^{\circ}$ of latitude at midnight. The radius of the northern hemisphere auroral oval during the interval 2000-2002 was determined using the method of circle-fitting developed by Milan et al. [2009a], applied to auroral images taken by the SI12 camera on the IMAGE spacecraft. Circles of increasing radius were centered on a point displaced from the pole toward the nightside by $5^{\circ}$ of magnetic latitude, and toward dusk by $2^{\circ}$. The mean brightness of the auroral image around the circumference of each circle was measured and the circle with the largest mean brightness was taken as the oval radius. This radius is accurate to $0.5^{\circ}$ of magnetic latitude, because each successive circle in the fitting process has a radius that is larger than the previous circle by $1^{\circ}$ of latitude. These measurements of the latitude of the proton auroral oval were compared with the latitude of the Heppner-Maynard Boundary determined simultaneously from 2 min intervals of SuperDARN data, provided the interval contained greater than 200 radar data points. The HMB, defined at midnight, was found to lie on average $2^{\circ}$ equatorward of the latitude of the proton auroral oval at midnight, which, according to a large statistical study by Boakes et al. [2008], lies $5^{\circ}$ equatorward of the OCB on average. The study also showed that the proton auroral oval and the HMB moved equatorward and poleward together, maintaining this offset during all but the largest and the smallest substorms. The measurement of the latitude of the HMB from the SuperDARN data is only accurate to $\pm 0.5^{\circ}$, and using the HMB as a proxy for the polar cap area is only advisable for large-scale statistical studies. In this study, we use changes in the monthly distribution of HMB latitudes as a proxy for changes in the average area of the polar cap over a solar cycle. We observe that the polar cap expanded to lower latitudes during a period of strong solar wind driving in the declining phase of the solar cycle, and contracted to higher latitudes during the recent solar minimum.

\section{Instrumentation}

\subsection{The SuperDARN Radar Network}

[8] The Super Dual Auroral Radar Network (SuperDARN) is traditionally understood as a network of radars located at high magnetic latitudes in the northern and southern hemispheres operating in the frequency range $8-20 \mathrm{MHz}$ [Greenwald, 1995; Chisham et al., 2007]. In the common operating mode, each radar sweeps through at least 16 look directions in either 60 or $120 \mathrm{~s}$, measuring the line-of-sight velocity of the ionospheric plasma using the Doppler-shift of the return signal. The amount of backscatter in a given field of view depends on many factors, including the opacity of the $D$ and $E$ regions of the ionosphere to the radio waves, and the occurrence of plasma irregularities in the $F$ region to generate the radar backscatter. These factors mean that although a region may be well covered by the radar fields of view, the amount of backscatter observed can be highly variable. The main array of SuperDARN radars became operational between 1993 and 2001, and the radars are located at high latitudes in the northern hemisphere, as shown in blue in Figure 2. The next phase of the SuperDARN array, the StormDARN radars, is shown in red. They are designed to measure ionospheric convection during particularly active geomagnetic conditions when the auroral oval is expanded, and so are located at lower geomagnetic latitudes. Finally the PolarDARN radars were constructed in 2007 and 2008 and are shown in green. By August 2012, there were 18 radars in operation in the northern hemisphere.

\subsubsection{The Map Potential Technique}

[9] The convection of ionospheric plasma at high latitudes is driven by an imposed electric field, generated largely by reconnection in the magnetosphere, and to a lesser extent viscous interaction between the magnetosheath flow and the magnetopause [e.g., Milan, 2004]. This imposed electric field 


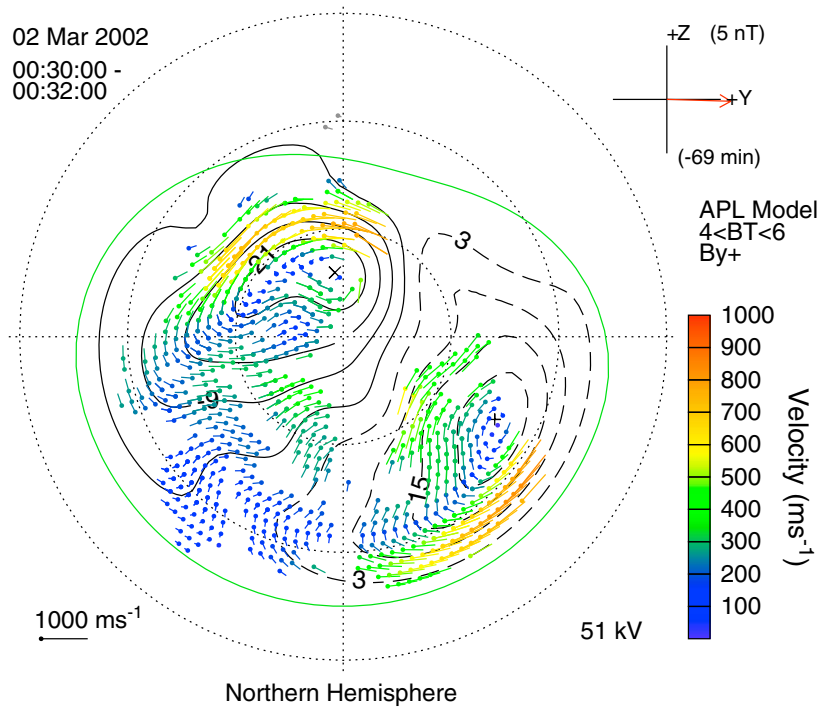

Figure 3. A SuperDARN potential map from 2 March 2002 at 00:30 UT. Noon is to the top of the figure and dusk to the left. The colored dots are the locations of radar observations, with the colour and the vector direction corresponding to the velocity of the flow given by the sixth-order spherical harmonic fit. The black lines are the ionospheric potential, and the coincident interplanetary magnetic field (IMF) data fed into the map potential model is shown at the top right. The solid green line is the HeppnerMaynard Boundary for this map, which crosses the midnight meridian at $65^{\circ}$.

is curl free and can be represented as the gradient of a potential, such that the contours of constant potential represent flow streamlines for the ionospheric plasma. The map potential technique [Ruohoniemi and Baker, 1998] is used to determine an ionospheric potential pattern within the northern hemisphere polar cap which best fits the SuperDARN data. This technique is a method of combining the line-of-sight radar data to give a global two-dimensional ionospheric convection pattern at $2 \mathrm{~min}$ cadence. The line-of-sight velocity data are gridded onto an equal area grid and the data in each square are averaged. In addition to the data within the current 2 min scan period, some temporal averaging of the data is performed, which involves taking all data from the preceding and following $2 \mathrm{~min}$ and folding it into the grid, with a $50 \%$ weighting for the data within the scan interval, and a $25 \%$ weighting for the data from the scans either side. An electrostatic potential function of the form of a spherical harmonic expansion is then fitted to this gridded data using a residual minimization technique to obtain the best fit. More details of the map potential technique can be found in Ruohoneimi and Baker [1998], Shepherd and Ruohoneimi [2000], Chisham and Pinnock [2002], Grocott et al. [2012], and references therein.

\subsubsection{The Heppner-Maynard Boundary}

[10] As discussed in section 1, the Heppner-Maynard Boundary (HMB) is the equatorward boundary of the ionospheric convection and is derived from the temporally averaged radar data assigned to a $2 \mathrm{~min}$ scan period, projected onto the SuperDARN equal area grid. The HMB is determined such that it is at the lowest latitude at which a minimum of three radar velocity measurements of greater than $100 \mathrm{~m} \mathrm{~s}^{-1}$ lie on the boundary, thus ensuring that the HMB encircles the main area of ionospheric convection. The shape of the HMB has been determined empirically such that even if there is a spatial gap in the radar coverage, the HMB can still be determined using radar data in the remaining sectors [e.g., Imber et al., 2013]. Its nonuniform shape means that in this paper the HMB latitude is defined as the intersection of the HMB with the midnight meridian, with a spatial resolution of $\pm 0.5^{\circ}$. The Heppner-Maynard boundary itself is used to scale the map potential statistical model to the size of the ionospheric convection pattern which is then added to the radar data in areas where there is no data coverage, to produce the global ionospheric potential map [Shepherd and Ruohoneimi, 2000]. A SuperDARN global convection map with good radar coverage is presented in Figure 3 from 00:30 UT on 2 March 2002 , in a magnetic local time-magnetic latitude grid with noon to the top of the Figure and dusk to the left. The colored dots are the location of SuperDARN measurements, and the color, length, and direction of the vectors are determined by the velocity of the flow at that location. The contours are the ionospheric potential given by the map potential technique and are a sixth-order spherical harmonic fit to the radar data. The green line is the HMB which clearly marks the equatorward edge of the radar scatter. The latitude of the HMB for this map, measured at midnight, is $65^{\circ}$. A black plus symbol marks the location of the most positive potential, and a black cross marks the location of the most negative potential according to the spherical harmonic fit to the data. The interplanetary magnetic field in the GSM Y-Z plane provided by the OMNI data set [King and Papitashvili, 2004] is shown at the top right of the Figure. The radar observations show an asymmetric twin cell convection pattern typical for intervals of southward and duskward IMF [e.g., Ruohoniemi and Greenwald, 1996], with a cross polar cap potential of $51 \mathrm{kV}$.

\subsection{Other Data Sets Used in This Study}

[11] The SuperMAG project combines data from over 300 magnetometer stations worldwide. The magnetometers measure perturbations in the horizontal component of the geomagnetic field, which are related to changes in the strength of the region 1 and 2 field-aligned currents and are a measure of the level of geomagnetic activity. The list of substorms used in this paper covering the interval 1996-2009 was identified using SuperMAG data and more information is presented in Newell and Gjerloev [2011].

\section{Observations}

[12] The latitude of the HMB at 24 MLT was calculated for each 2 min SuperDARN map containing greater than 150 SuperDARN data points from 1 January 1996 until 31 August 2012. The threshold of 150 vectors is slightly different from the one used in our previous paper [Imber et al., 2013]. We have changed the threshold from 200 vectors per map to 150 vectors as we have found that the lower threshold is an optimum value for providing us with large statistics with little impact on the ability of the maps to determine the HMB.

[13] The occurrence distribution of the HMB latitude was generated for each month within this time period. The number of maps available per month varied by a factor of more than 30 due to seasonal and solar cycle effects. In order 

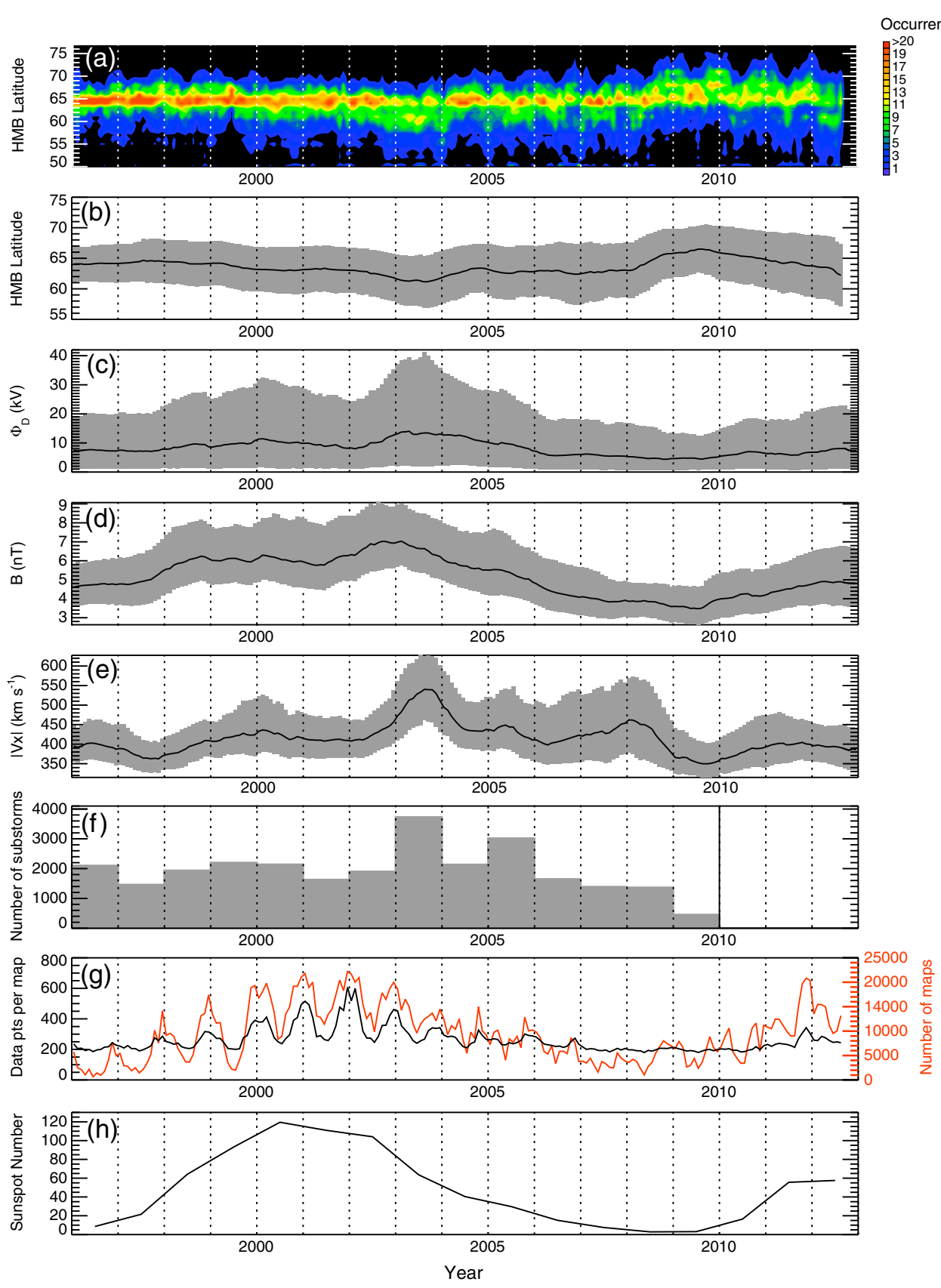

Figure 4. (a) Monthly occurrence distributions of the HMB latitude from SuperDARN radar observations during the time period January 1996 to August 2012. The contours are the normalized occurrence according to the color bar on the right of the panel. (b) Mean and standard deviation of the distribution of HMB latitudes over a 12 month sliding window. The same sliding 12 month window is applied to Figures $4 \mathrm{c}-4 \mathrm{e}$, which display the median and quartiles of the distributions of (c) the coupling function derived by Milan et al. [2012], (d) the 1 min resolution OMNI solar wind magnetic field magnitude, and (e) the OMNI solar wind velocity in the GSM X direction. (f) A histogram of the number of substorms per year identified using the SuperMAG data. (g) The average number of data points per SuperDARN map (black) and the average number of SuperDARN maps included in the study (red), per month. (h) The yearly sunspot number provided by the Solar Influences Data Analysis Center.

to be able to compare data across this time period, the HMB occurrence distributions were normalized and are presented in Figure 4a. The colored contours are the normalized occurrence distribution at $1^{\circ}$ resolution between 50 and $75^{\circ}$ latitude. The dashed white lines mark the start of each year. The distributions appear to consistently peak around $64^{\circ}$ throughout the interval. The only large variations from this are during 2003 when a secondary peak appears at $61^{\circ}$ and between mid-2008 and mid-2011 when a second peak appears at $68^{\circ}$. The mean HMB latitude determined from a sliding 12 month window of data is displayed in Figure $4 \mathrm{~b}$. The grey-shaded area spans \pm 1 standard deviation of the mean. 
[14] The same sliding 12 month window is applied to Figures $4 \mathrm{c}-4 \mathrm{e}$, which display the median and quartiles of the distributions of (c) the coupling function derived by Milan et al. [2012], (d) the $1 \mathrm{~min}$ resolution OMNI solar wind magnetic field magnitude, and (e) the OMNI solar wind velocity in the GSM X direction. The IMF strength gradually increased from $5 \mathrm{nT}$ in 1996 to $\sim \mathrm{nT}$ in 2002 and then decreased to a minimum of $4 \mathrm{nT}$ in 2009. The solar wind speed consistently averaged $400-450 \mathrm{~km} \mathrm{~s}^{-1}$ for the majority of the interval, with a clear enhancement to $550 \mathrm{~km} \mathrm{~s}^{-1}$ during 2003 and a decrease to $350 \mathrm{~km} \mathrm{~s}^{-1}$ during 2009.

[15] The number of substorms observed per year between 1996 and 2009 is shown as a histogram in Figure 4f. This list was determined by Newell and Gjerloev [2011] using SuperMAG data and shows a solar cycle dependence, with the highest number of substorms during the declining phase of the solar cycle. This dependence was earlier identified by Tanskanen [2009] using ground-based magnetometer data from the Scandinavian IMAGE network.

[16] Figure 4g shows a monthly average of the number of SuperDARN data points per map in black and the number of maps included for each month of the study in red. Each map corresponds to a 2 min interval, so the maximum number of maps that can be included in a month is over 22,000. There is an annual cycle as well as the 11 year solar cycle visible in both the number of data points per map and the total number of maps.

[17] The yearly sunspot number in Figure $4 \mathrm{~h}$ is a measure of the 11 year solar activity cycle and was provided by the Solar Influences Data Analysis Centre [SIDC team, 2012]. Year 1996 marked the beginning of solar cycle 23, characterized by a minimum in the sunspot number. The maximum of solar cycle 23 was observed during the years 2000-2002, with the next minimum spanning 2007-2010. Solar cycle 23 was unusual, however, as the solar minimum at the end of the cycle was unusually deep and extremely extended relative to previous cycles.

\section{Discussion}

[18] The HMB latitude distributions displayed in Figure 4a covering the years 1996-2002 are remarkably constant, with a narrow peak value of $64^{\circ}$ magnetic latitude. The HMB is rarely measured below $57^{\circ}$ or above $70^{\circ}$ during this time period. The average number of data points per map shown in Figure 4g varies by up to a factor of three during each year. Despite this, the HMB latitude distributions do not vary significantly on a month-by-month basis, suggesting that our minimum threshold of 150 SuperDARN data points per map is sufficient to accurately determine the latitude of the HMB.

[19] While the monthly HMB latitude distributions are very stable during the early part of the study, the interval beginning in mid-2002 until mid-2004 appears to show quite a different characteristic distribution. Instead of a narrow distribution with a single peak at $64^{\circ}$, the monthly latitude distributions are much broader, extending to lower latitudes than previously. In addition, there appears to be a second peak in the distribution during 2003 at $61^{\circ}$. Following this unusual period, the distribution narrows once again and returns to a distribution similar to that prior to 2002, remaining relatively constant until 2008. During the interval mid-2008 to mid-2011, the HMB latitude distributions again broaden, and at times a second peak is observed at higher latitudes than the persistent peak at $64^{\circ}$ observed throughout the 17 years of study.

\subsection{Instrumental Considerations}

[20] The year-on-year expansion of the SuperDARN radar network is shown in Figure 2a. Changes in the instrumentation must be considered when studying long-term trends using SuperDARN radar data. The addition of radars at lower latitudes would be expected to increase the number of maps per month included in the study as there is increased likelihood of the number of data points per map exceeding the 150 data point threshold. It may also increase the probability that the HMB is observed at lower latitudes as the radar coverage is increased in this area.

[21] The short-term nature of the equatorward shift of the HMB during 2002-2004 makes instrumental effects an unlikely cause. No new radars were constructed during this time to influence the determination of the HMB. The Kodiak and King Salmon radars were built in 2000 and 2001, respectively. These radars were at slightly lower latitudes than the previous northern hemisphere radars and if these were the cause of the change in HMB distribution they would have had much more of an immediate impact on the observations in Figure 4a. There was also no immediate change in the number of maps included in the study, or the number of data points per map shown in Figure $4 \mathrm{~g}$ following the addition of these radars to the network. Similarly the return of the HMB latitude distribution to higher latitudes during early 2004 cannot be attributed to instrumental effects as the number of radars did not change between 2001 and 2005.

[22] Between 2007 and 2011, several new radars were commissioned. Three of these radars, Wallops Island, Hokkaido, and Blackstone, were part of the mid-latitude StormDARN radar network, designed to observe the lowerlatitude portion of the ionospheric convection pattern when it extends equatorward beyond the range of the higher latitude radars. These radars did not observe a great deal of scatter during their early years and, due to their location, could not have produced the poleward shift in HMB latitude observed during this time period.

[23] Perhaps of more importance to this study were two high latitude radars, Rankin Inlet and Inuvik, brought into operation during 2007 and 2008, respectively. These radars observed more backscatter than those at lower latitudes during their early years and therefore could have been responsible for the appearance of the second higher latitude peak in the HMB distributions, given the 150 data point threshold for each map. Rankin Inlet did not appear to have any instant impact on the HMB distribution in 2007. Inuvik came online in June 2008, which coincided with the appearance of the second, poleward peak in HMB latitude distribution, and cannot be ruled out as a cause of the shift. We note that the interval is not just dominated by the appearance of a secondary peak at higher latitudes, but that the entire envelope of the distribution also shifts poleward. This demonstrates that the existing radars in the main array are not observing as much backscatter as during earlier years, which would be expected if the statistical location of the OCB was shifted toward higher latitudes. 
[24] The uncertainty brought about by the addition of the PolarDARN radars is whether the appearance of a higher latitude secondary peak in the HMB distributions during 2008 may have taken place earlier than observed, but the instrumentation was not available to measure that shift until both PolarDARN radars were operational. The arrival of the secondary peak at high latitudes in mid-2008 was delayed by $\sim 12$ months relative to the start of the extended solar minimum, suggesting that this may have been the case.

[25] The distribution of HMB latitudes during 2011 and 2012 shows clear signs of a return to a distribution similar to those observed prior to 2008 , with even a suggestion of a secondary peak at $61^{\circ}$ during 2012 . The disappearance of this second high latitude peak during 2011 again indicates that its existence was not due to instrumental effects. We suggest that during the second half of 2011 and 2012 the OCB was statistically more likely to be observed at lower latitudes than during the preceding years, such that the radars in the main array started to observe more backscatter, bringing the statistical location of the HMB to lower latitudes.

\subsection{Solar Cycle Variations}

[26] The interval January 1996-August 2012 extends just over one solar cycle. Solar cycle 23 was unusual, characterized by a peak during the year 2000 and a solar minimum spanning the interval 2007-2010 as shown by the sunspot number presented in Figure $4 \mathrm{~h}$. The extremely deep and extended solar minimum has produced solar wind conditions that are the quietest in recent decades, and this has provided a unique opportunity to observe the Earth's magnetosphere in its base state.

[27] The solar wind conditions vary significantly depending on the solar cycle, and the median and quartiles of the distributions of the interplanetary magnetic field (IMF) strength and the solar wind velocity (in the GSM X direction) measured with a 12 month sliding window are displayed in Figures $4 \mathrm{~d}$ and $4 \mathrm{e}$. The IMF strength has a broad peak during solar maximum, while the solar wind speed is more stable around $400-450 \mathrm{~km} / \mathrm{s}$, with a significant but short-lived increase during 2003 to a value of $\sim 550 \mathrm{~km} / \mathrm{s}$, and a decrease in 2009 and 2010 to well below $400 \mathrm{~km} / \mathrm{s}$. We suggest that the unusual distributions of HMB latitude during 2003 and more recently 2008-2011 could be related to changes in the properties of the solar wind as these are known to control the rate of solar wind-magnetosphere coupling. The OMNI solar wind data were used to calculate the solar wind-magnetosphere coupling function derived by Milan et al. [2012], given in equation (3). The median and quartiles of this coupling function over a 12 month sliding window are presented in Figure 4c. The level of solar wind-magnetosphere coupling predicted by the coupling function in 2003 is significantly higher than for any other year covered by this study, and Figure 4f shows that there were significantly more substorms during this year, too, suggesting that the level of dayside and tail reconnection was higher. Conversely during the interval 2007-2010, the coupling function in Figure $4 \mathrm{c}$ predicts an unusually low level of solar wind-magnetosphere coupling, and there were very few substorms.

[28] To summarize, the data in Figure 4 show that the HMB distribution developed a second, lower-latitude peak during 2003, a year of enhanced solar wind-magnetosphere driving and therefore intense and frequent substorms [Tanskanen, 2009]. During the solar minimum, 2007-2010, a very quiet period in terms of geomagnetic activity and with low solar wind forcing, a secondary peak in the HMB distribution was observed at higher latitudes. The exact timing of the appearance of this peak may be due to the Inuvik radar becoming operational; however, it is clear that during late 2011 and 2012, the distribution returned to a lower-latitude distribution, suggesting that the secondary peak observed was not due to instrumental effects.

[29] The advantage of using the HMB as a proxy for the size of the polar cap is that the SuperDARN radars have reliably provided HMB latitudes at 2 min cadence for the last 17 years, while similar studies using auroral images are limited by orbital viewing constraints such that continuous coverage is not possible. Further, global auroral images have not been available since 2008, so it has not been possible to use these to characterize the response of the polar cap to the extremely quiet solar minimum. This is the first study to observe the long-term variations in the polar cap area over the course of an entire solar cycle. Unfortunately we are unable to directly compare the HMB distributions of the most recent solar minimum with the previous minimum in 1995/1996 due to the dramatic change in instrumentation during the intervening years.

\subsection{Solar Cycle Influences on SuperDARN Data Coverage}

[30] The average number of data points per SuperDARN map included in the study is shown in Figure 4g (all maps with less than 150 data points are excluded). At the start and end of the interval, the average number of data points per map is 200; however, during December 2001 this reaches 606 data points. There is a clear annual variation during the years either side of solar maximum, with up to three times more data points observed during northern hemisphere winter than during the summer. This variation is thought to be largely due to a combination of higher $F$ region density in winter, more uniform $F$ region density in summer and higher $D$ region absorption in summer [e.g., Milan et al., 1997]. These factors all combine to create more favorable radar propagation paths and more backscatter targets in northern hemisphere winter. This variation is barely observed during the first solar minimum and is not observed at all during the most recent, extended solar minimum.

[31] The number of SuperDARN maps included in the study is shown in Figure $4 \mathrm{~g}$ as the red line, which rises and falls with the number of data points per map, as expected. The number of maps per month included in the study ranges from $\sim 650$ to over 20,000 . It appears to be the same, if not higher, during the intervals 2003 and 2008-2010 than the surrounding months; therefore, we do not believe that the unusual HMB distributions during these periods are due to poor statistics. The number of maps per month containing greater than 150 data points would be expected to rise year upon year as the number of radars increases and the spatial coverage of the polar cap improves. It is interesting to observe that despite the increased spatial coverage, the number of maps and the number of data points per map follow the solar activity cycle very closely. We intend to investigate this further in a future study. 


\section{Conclusions}

[32] The HMB encircles the ionospheric convection pattern at its lowest latitude and is expected to expand and contract at approximately the same rate as the polar cap due to reconnection opening or closing magnetic flux. The unusually low latitude of the HMB distribution during 2003 coincides with a period of strong dayside driving and frequent and intense substorm activity, which is characterized by large expansions and sudden contractions of the polar cap. The period of high latitude HMB distributions during 2008-2010 coincides with the deep, extended solar minimum, characterized by extremely weak dayside driving and very low geomagnetic activity levels. The changes in the HMB distributions during these two periods of unusual solar wind driving therefore directly reflect the state of the magnetosphere over monthly averaged time scales. We have used the HMB to study the distribution of polar cap sizes over an entire solar cycle, something not achievable using in situ plasma measurements or auroral observations, and have observed the global magnetospheric response to the quietest solar minimum in over a century. We suggest that the HMB is a valuable proxy for large statistical studies investigating the state of the magnetosphere when studying long-term trends in the Sun-Earth system.

[33] Acknowledgments. We gratefully acknowledge CDAWeb and the Solar Influences Data Analysis Centre for providing the OMNI data and the list of sunspot numbers used in this study. For the substorm list, we acknowledge the SuperMAG initiative and the SuperMAG collaborators. SuperDARN is a collection of radars funded by national scientific funding agencies of Australia, Canada, China, France, Japan, South Africa, the United Kingdom, and the United States of America, and the authors gratefully acknowledge the use of SuperDARN data. The SuperDARN data used in this study were processed as part of the European Cluster Assimilation Technology (ECLAT) project. The research leading to these results has received funding from the European Union Seventh Framework Programme (FP7/2007-2013) under grant agreement 263325.

[34] Robert Lysak thanks Gareth Chisham and another reviewer for their assistance in evaluating this paper.

\section{References}

Akasofu, S.-I. (1964), The development of the auroral substorm, Planet. Space Sci., 12, 273-282.

Boakes, P. D., S. E. Milan, G. A. Abel, M. P. Freeman, G. Chisham, B. Hubert, and T. Sotirelis (2008), On the use of IMAGE FUV for estimating the latitude of the open/closed magnetic field line boundary in the ionosphere, Ann. Geophys., 26, 2759-2769, doi:10.5194/angeo-26-2759-2008.

Boakes, P. D., S. E. Milan, G. A. Abel, M. P. Freeman, G. Chisham, and B. Hubert (2009), A statistical study of the open magnetic flux content of the magnetosphere at the time of substorm onset, Geophys. Res. Lett., 36, L04105, doi:10.1029/2008GL037059.

Borovsky, J. E. (2008), The rudiments of a theory of solar wind/magnetosphere coupling derived from first principles, J. Geophys. Res., 113, A08228, doi:10.1029/2007JA012646.

Chisham, G., and M. Pinnock (2002), Assessing the contamination of SuperDARN global convection maps by non-F-region backscatter, Ann. Geophys., 20, 13-28.

Chisham, G., et al. (2007), A decade of the Super Dual Auroral Radar Network (SuperDARN): Scientific achievements, new techniques and future directions, Surv. Geophys., 28, 33-109, doi:10.1007/s10712-007-9017-8.

Cowley, S. W. H., and M. Lockwood (1992), Excitation and decay of solar wind-driven flows in the magnetosphere-ionosphere system, Ann. Geophys., 10, 103-115.

Dungey, J. W. (1961), Interplanetary magnetic fields and the auroral zones, Phys. Rev. Lett., 6, 47-48.

Greenwald, R. A. (1995), DARN/SuperDARN: a global view of the dynamics of high-latitude convection, Space Sci. Rev., 71, 761-796.

Grocott, A., S. E. Milan, S. M. Imber, M. Lester, and T. K. Yeoman (2012), A quantitative deconstruction of the morphology of high-latitude ionospheric convection, J. Geophys. Res., 117, A05317, doi:10.1029/ 2012JA017580.
Heppner, J. P. (1977), Empirical models of high-latitude electric fields, J. Geophys. Res., 82, 1115-1125.

Heppner, J. P., and N. C. Maynard (1987), Empirical high-latitude electric field models, J. Geophys. Res., 92(A5), 4467-4489, doi:10.1029/ JA092iA05p04467.

Hones, E. W., Jr. (1979), Plasma flow in the magnetotail and its implications for substorm theories, in Dynamics of the Magnetosphere, edited by S.-I. Akasofu, 545 pp., D. Reidel, Dordrecht, The Netherlands.

Hubert, B., S. E. Milan, A. Grocott, C. Blockx, S. W. H. Cowley, and J. C. Gerard (2006), Dayside and nightside reconnection rates inferred from IMAGE FUV and Super Dual Auroral Radar Network data, J. Geophys. Res., 111, A03217, doi:10.1029/2005JA011140.

Hubert, B., A. T. Aikio, O. Amm, T. Pitkänen, K. Kauristie, S. E. Milan, S. W. H. Cowley, and J.-C. Gérard (2010), Comparison of the open-closed field line boundary location inferred using IMAGE-FUV SI12 images and EISCAT radar observations, Ann. Geophys., 28, 883-892, doi:10.5194/ angeo-28-883.

Imber, S. M., S. E. Milan, and M. Lester (2013), The Heppner-Maynard Boundary measured by SuperDARN as a proxy for the latitude of the auroral oval, J. Geophys. Res. Space Physics, 118, 685-697, doi:10.1029/ 2012JA018222.

Kan, J. R., and L. C. Lee (1979), Energy coupling function and solar wind magnetosphere dynamo, Geophys. Res. Lett., 6, 577-580.

King, J. H., and N. E. Papitashvili (2004), Solar wind spatial scales in and comparisons of hourly Wind and ACE plasma and magnetic field data, J. Geophys. Res., 110, A02209, doi:10.1029/2004JA010804.

Lockwood, M., and Cowley, S. W. H. (1992), Ionospheric convection and the substorm cycle, in Proceedings of the International Conference on Substorms (ICS-1), Eur. Space Agency Spec. Publ., ESP SP-335, pp. 99-109.

Longden, N., G. Chisham, M. P. Freeman, G. A. Abel, and T. Sotirelis (2010), Estimating the location of the open-closed magnetic field line boundary from auroral images, Ann. Geophys., 28, 1659-1678, doi:10.5194/angeo-28-1659-2010.

McPherron, R. L., C. T. Russell, and M. P. Aubry (1973), Satellite studies of magnetospheric substorms on August 15, 1968, J. Geophys. Res., 78(16), 3131-3149.

Milan, S. E. (2004), Dayside and nightside contributions to the cross polar cap potential: Placing an upper limit on a viscous-like interaction, Ann. Geophys., 22, 3771-3777, doi:10.5194/angeo-22-3771-2004.

Milan, S. E., T. K. Yeoman, M. Lester, E. C. Thomas, and T. B. Jones (1997), Initial backscatter occurrence statistics from the CUTLASS HF radars, Ann. Geophys., 15, 703-718, doi:10.1007/s00585-997-0703-0.

Milan, S. E., S. W. H. Cowley, M. Lester, D. M. Wright, J. A. Slavin, M. Fillingim, C. W. Carlson, and H. J. Singer (2004), Response of the magnetotail to changes in the open flux content of the magnetosphere, J. Geophys. Res., 109, A04220, doi:10.1029/2003JA010350.

Milan, S. E., G. Provan, and B. Hubert (2007), Magnetic flux transport in the Dungey cycle: A survey of dayside and nightside reconnection rates, J. Geophys. Res., 112, A01209, doi:10.1029/2006JA011642.

Milan, S. E., P. D. Boakes, and B. Hubert (2008), Response of the expanding/contracting polar cap to weak and strong solar wind driving: implications for substorm onset, J. Geophys. Res., 113, A09215, doi:10.1029/2008JA013340.

Milan, S. E., J. Hutchinson, P. D. Boakes, and B. Hubert (2009a), Influences on the radius of the auroral oval, Ann. Geophys., 27, 2913-2924.

Milan, S. E., A. Grocott, C. Forsyth, S. M. Imber, P. D. Boakes, and B. Hubert (2009b), A superposed epoch analysis of auroral evolution during substorm growth, onset and recovery: open magnetic flux control of substorm intensity, Ann. Geophys., 27, 659-668.

Milan, S. E., J. S. Gosling, and B. Hubert (2012), Relationship between interplanetary parameters and the magnetopause reconnection rate quantified from observations of the expanding polar cap, J. Geophys. Res., 117, A03226, doi:10.1029/2011JA017082.

Newell, P. T., and J. W. Gjerloev (2011), Evaluation of SuperMAG auroral electrojet indices as indicators of substorms and auroral power, J. Geophys. Res., 116, A12211, doi:10.1029/2011JA016779.

Newell, P. T., S. Wing, C. I. Meng, and V. Sigillito (1991), The auroral oval position, structure and intensity of precipitation from 1984 onward: an automated on-line data base, J. Geophys. Res., 96, 5877-5882.

Newell, P. T., T. Sotirelis, K. Liou, C.-I. Meng, and F. J. Rich (2007), A nearly universal solar wind-magnetosphere coupling function inferred from 10 magnetospheric state variables, J. Geophys. Res., 112, A01206, doi:10.1029/2006JA012015.

Østgaard, N., S. B. Mende, H. U. Frey, J. B. Sigwarth, A. Åsnes, and J. M. Weygand (2007), Auroral conjugacy studies based on global imaging, J. Atmos. Sol.-Terr. Phys., 69(3), 249-255, ISSN:13646826, doi:10.1016/j.jastp.2006.05.026.

Pulkkinen, T. I., D. N. Baker, D. H. Fairfield, R. J. Pellinen, J. S. Murphree, R. D. Elphinstone, R. L. McPherron, J. F. Fennell, R. E. Lopez, and T. Nagai (1991), Modeling the growth phase of a substorm using the 


\section{IMBER ET AL.: SOLAR CYCLE VARIATIONS IN POLAR CAP AREA}

Tsyganenko model and multi-spacecraft observations: CDAW-9, Geophys. Res. Lett., 18(11), 1963-1966.

Ruohoniemi, J. M., and K. B. Baker (1998), Large-scale imaging of high-latitude convection with Super Dual Auroral Radar Network HF radar observations, J. Geophys. Res., 103(A9), 20,797-20,811, doi:10.1029/98JA01288.

Ruohoniemi, M., and R. A. Greenwald (1996), Statistical patterns of high latitude convection obtained from Goose Bay HF radar observations, J. Geophys. Res., 101(21), 743-21,763.

Shepherd, S. G., and J. M. Ruohoniemi (2000), Electrostatic potential patterns in the high-latitude ionosphere constrained by SuperDARN measurements, J. Geophys. Res., 105(A10), 23,005-23,014, doi:10.1029/2000JA000171.

SIDC team (2012), Monthly Report on the International Sunspot Number, 1995-2012, http://www.sidc.be/sunspot-data/, World Data Cent. for Sunspot Index, Royal Observatory of Belgium.
Siscoe, G. L., and T. S. Huang (1985), Polar cap inflation and deflation, J. Geophys. Res., 90(A1), 543-547.

Siscoe, G. L., N. U. Crooker, and K. D. Siebert (2002), Transpolar potential saturation: Roles of region 1 current system and solar wind ram pressure, J. Geophys. Res., 107(A10), 1321, doi:10.1029/ 2001JA009176.

Tanskanen, E. I. (2009), A comprehensive high-throughput analysis of substorms observed by IMAGE magnetometer network: Years 19932003 examined, J. Geophys. Res., 114, A05204, doi:10.1029/ 2008JA013682.

Taylor, J. R., T. K. Yeoman, M. Lester, B. A. Emery, and D. J. Knipp (1996), Variations in the polar cap area during intervals of substorm activity on March 20-21 1990 deduced from AMIE convection patterns, Ann. Geophys., 14, 879-887. 